\title{
Parasitic oscillations in smooth-wall circular symmetric gyrotron beam ducts
}

\author{
J. Genoud · S. Alberti · T. M. Tran · G. Le \\ Bars · P. Kaminski · J-Ph. Hogge · K. A. \\ Avramidis - M. Q. Tran
}

Received: date / Accepted: date

\begin{abstract}
In order to study parasitic oscillation that may occur in a realistic beam duct upstream to the gyrotron cavity, the self-consistent linear and spectral code TWANGlinspec has been modified. The large inhomogeneities in the smooth-wall beam duct geometry or in the magnetic field profile required the implementation of a numerical approach using a hybrid finite element method. The new model permits to characterize a large number of potentially spurious TE modes. Compared to previous studies on gyrotron beam duct instabilities, an extended interaction space including also the gyrotron cavity has been considered. The role of the connecting part between the beam duct and the cavity, called spacer, is highlighted and it is shown that the gyro backward-wave TE modes excited in this region generally have their minimum starting current. The sensitivity of the minimum starting current on electron beam velocity spread is also evaluated.
\end{abstract}

\section{Introduction}

Parasitic oscillations are one of the main point hindering high power gyrotrons operation for fusion applications. These oscillations have been observed in high power gyrotrons and their generation is commonly attributed to a beam-wave

J. Genoud · S. Alberti · T. M. Tran · G. Le Bars · P. Kaminski · J-Ph. Hogge · M. Q. Tran Ecole Polytechnique Fédérale de Lausanne (EPFL),

Swiss Plasma Center (SPC),

$\mathrm{CH}-1015$, Lausanne, Switzerland

T.M. Tran died before publication of this work was completed.

K. A. Avramidis

Institute for Pulsed Power and Microwave Technology,

Karlsruhe Institue of Technology,

76131 Karlsruhe Germany 
interaction in the gyrotron beam-duct situated upstream to the cavity. The instability responsible for the excitation of the beam-duct parasitic oscillations is generally a gyro-backward wave. If excited, it introduces significant electron beam velocity and energy spreads inducing a significant degradation of the gyrotron interaction efficiency.

A notable effort has been devoted to the theoretical and experimental study of these oscillations. Based on these studies [1,2,3,4], essentially three different strategies for suppressing spurious instabilities in gyrotron beam ducts have been studied and implemented. The first one is to use a smooth-wall dielectric loaded beam duct, made from SiC [2]. The second is the use of a metallic beam duct with a random surface $[1,5]$. The third one is the stacked ring beam duct, composed by rings of copper and dielectric material, like BeOSiC $[4,6]$. In [4], indented copper rings are introduced in order to reduce the parasitic oscillations of beam ducts with smooth-wall copper rings. Despite this effort, gyrotrons with stacked rings beam-tunnel have often experienced parasitic oscillations.

Theoretically, various models exist. A distinction can be made between the models treating the beam-wave interaction self-consistently or not. Among the non-self consistent models, some are assuming a fixed field profile [1]. They compute the electric field profile for a given geometry and evaluate afterwards its effect on the interaction [7]. When dielectric loadings are used, usually their effect is based on non-self-consistent models [8]. For stacked rings beam ducts, the extremely complicated electromagnetic system does not allow a self consistent model so far $[7,9]$. Another approach to approximate a dielectric loading is to use a metallic boundary condition with a very low conductivity $[10,11,12]$. The less restrictive approach is a self-consistent approach considering the correct boundary condition in the presence of the dielectric coating [13].

The need of a self-consistent code for parasitic oscillations is generally accepted, as the backward wave nature involves strong self-consistent effects. This paper presents a self-consistent linear model developed to simulate parasitic oscillation in smooth-wall metallic or dielectric loaded beam ducts. The model is applied to simulate an extended region including a smooth-wall approximation of a beam duct, the spacer (connecting part between the spacer and the cavity) and the cavity. The geometry is based on the dual frequency gyrotron at the Swiss Plasma Center (SPC) $[14,15]$. The exact geometry is considered for the spacer and cavity regions while a smooth-wall envelope approximation is considered for the stacked ring beam duct. The need of considering the extended region is presented. In order to account for the large inhomogeneities in the beam duct region, a new numerical method based on a hybrid finite element scheme is implemented. This model is presented in section II. In Section III, some systematic parametric studies examples are shown. In Section IV, a realistic beam duct situation is treated first linearly and then with a nonlinear model also including velocity spread effects. Finally in V, a distributed losses study is presented. Section $\mathrm{V}$ concludes the paper. 


\section{Model}

The models used to study the parasitic oscillations in smooth-wall metallic beam ducts are derived from the monomode nonlinear, self-consistent TWANG model [16], based on the common slow time scale formulation. Two complementary self-consistent codes are used, TWANGlinspec [17] and TWANG-PIC [23]. The first one is a linear and spectral model [17]. The linearization has been done considering a moment-approach [18], allowing to reduce the system of $\mathrm{N}+1$ equations, $\mathrm{N}$ equations for the $\mathrm{N}$ electrons and one wave equation, to a system of three Partial Differential Equations for three moments. In the linear model, the only additional assumption made is to consider the perturbed parallel electron momentum to be constant in time, which implies that the self-consistent magnetic field is neglected. It is important to stress that the momentum approach used in this paper does not allow to treat effects associated to electron beam velocity and or energy spread. The details of the derivation as well as the numerical discretization can be found in $[17,18]$. In [17], the model has been validated by comparing its results with experimental measurements involving high and low power gyrotrons. The aim of this paper is to extend the model to the beam duct region. Originally, the model is already well suited for this purpose as it takes into account all the spatial inhomogeneities, such as the cavity wall tapering, the magnetic field profile or the wall losses. Indeed, unlike for the cavity case, these inhomogeneities are much larger for the beam duct, leading to longitudinal dependency for most of the physical parameters. In figure 1 is shown a longitudinal cross section of an "extended" geometry including the cavity, the usual interaction zone, the spacer and the beam duct. Nevertheless, as will be explained later, for taking into account the large inhomogeneities compared to [17], a new numerical scheme based on hybrid finite elements has been implemented.

The equations in their linear and spectral formulation are recalled here [17]:

$$
\begin{aligned}
i \Omega \pi_{1} & =\frac{\mathrm{d}}{\mathrm{d} \hat{z}}\left(\pi_{1} \beta_{z}\right)-A_{1} \pi_{1}-i C_{1} \pi_{2}+C_{2} C_{0} F \\
i \Omega \pi_{2} & =\frac{\mathrm{d}}{\mathrm{d} \hat{z}}\left(\pi_{2} \beta_{z}\right)-A_{2} \pi_{2}+i C_{1} \pi_{1} \\
-2 \Omega F & =\frac{\mathrm{d}^{2} F}{\mathrm{~d} \hat{z}^{2}}+\kappa_{\| 0}^{2} F-i C_{3} C_{0} \pi_{1} .
\end{aligned}
$$

The two first ordinary differential equations (1a) and (1b) describe the electron motion while (1c) is the wave equation. $\pi_{1}(\hat{z}, \Omega)=\left\langle\mathcal{P}_{1}^{*}\right\rangle, \pi_{2}(\hat{z}, \Omega)=\left\langle\mathcal{P}_{1} e^{2 i \psi_{0}}\right\rangle$ are moments of the linearized perturbation of the electron perpendicular momenta $\mathcal{P}_{1}$ and $\psi_{0}$ is the slow time scale electron phase at equilibrium. $F(\hat{z}, \Omega)=$ $\frac{e}{m_{e} c^{2}} \frac{s^{s}}{2^{s} s !} \frac{E}{k_{\perp}}$ is the normalized rf-field envelope. $\beta_{z}$ is the electron longitudinal ve-

locity. The two variables are the normalized axial position $\hat{z}=\frac{\omega_{0}}{c} z$, with $\omega_{0}$ a reference frequency and $c$ the speed of light, and $\Omega$ a complex term representing the frequency mismatch with respect to the reference frequency $\omega_{0}$ :

$$
\omega_{\mathrm{rf}}=\omega_{0}(1+\Omega) .
$$




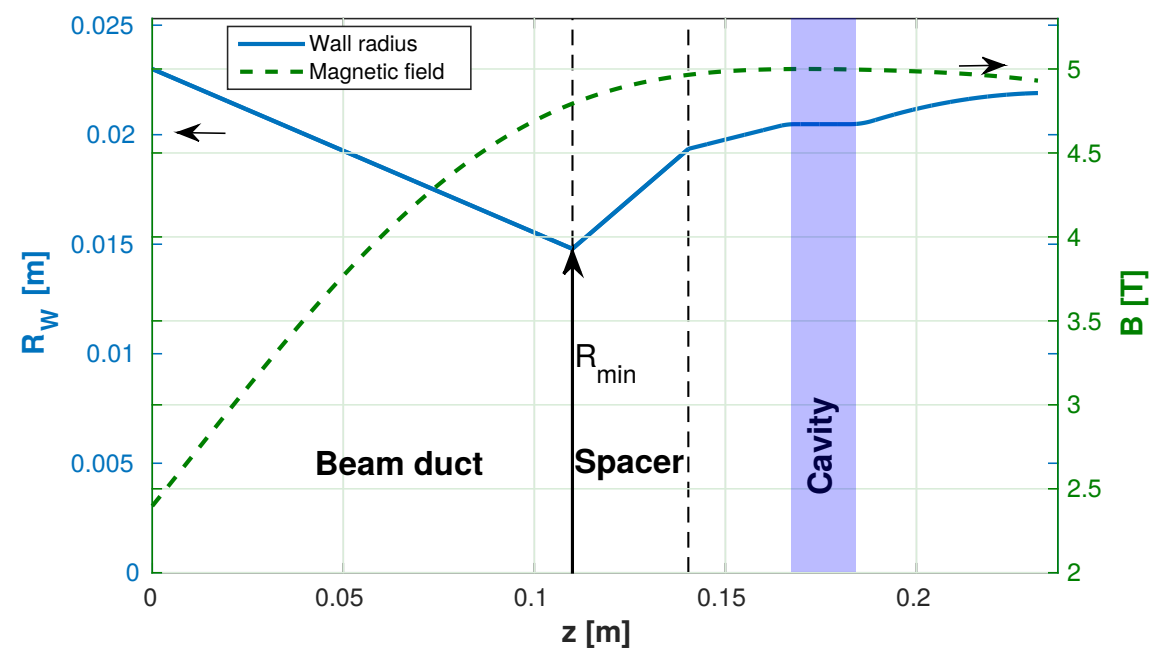

Fig. 1 Example of geometry considered. The usual interaction zone considered is the cavity, the constant radius part highlighted in blue, and its uptaper and downtaper. In this work, this zone is extended to the beam duct and spacer region.

Consistently with the wave envelope approximation, $\left|\operatorname{Re}(\Omega) / \omega_{0}\right| \ll 1$ is assumed. The other quantities are :

$$
\begin{aligned}
& A_{1}=i \Delta_{0}+i C_{1}+\beta_{z} \delta \\
& A_{2}=i \Delta_{0}-i C_{1}+\beta_{z} \delta \\
& C_{0}=\left(\frac{k_{\perp}}{k_{0}}\right)^{s}\left(\frac{s \Omega_{c}}{\omega_{0}}\right)^{1-s} J_{m-s}\left(k_{\perp} R_{g}\right), \\
& C_{1}=\frac{p_{\perp 0}^{2}}{2 s \gamma_{0}^{2}} \\
& C_{2}=s p_{\perp 0}^{2 s-2}, \\
& C_{3}=\frac{e Z_{0}}{m_{e} c^{2} p_{z 0}} \frac{I_{b}}{C_{m p}}\left(\frac{s^{s}}{2^{s} s !}\right)^{2}, \\
& \Delta_{0}=1-\frac{s \Omega_{c}}{\gamma_{0} \omega_{0}}, \\
& \kappa_{\| 0}^{2}=1-\frac{k_{\perp}^{2}}{k_{0}^{2}}\left[1-\frac{\delta_{\text {eff }}}{R_{w}}\right] .
\end{aligned}
$$

All these variables are equilibrium quantities. In this model, the Ohmic dissipation due to a metallic boundary condition is taken into account via a surface 
impedance boundary term correcting the parallel wave number $\kappa_{\| 0}$, with

$$
\delta_{\text {eff }}=(1+i)\left(1+\frac{m^{2}}{\nu_{m p}^{2}-m^{2}} \frac{k_{0}^{2}}{k_{\perp}^{2}}\right) \delta_{s k} .
$$

In these expressions $k_{0}, k_{\|}$and $k_{\perp}=\nu_{m p} / R_{w}$ are respectively the wave number and its parallel and perpendicular components. $m$ and $p$ are the azimuthal and radial mode numbers for a transverse mode $T E_{m, p} . \nu_{m p}$ is the $\mathrm{p}^{\text {th }}$ root of the derivative of the bessel function $J_{m}$ and $R_{w}$ is the wall radius. $\delta_{s k}=\sqrt{\frac{2}{\omega_{0} \mu_{0} \sigma}}$ is the skin depth, with $\mu_{0}$ the vacuum permeability and $\sigma$ the wall conductivity. Other quantities are: $\Omega_{c}$ the cyclotron angular frequency, $s$ the cyclotron resonance harmonic number, $p_{\perp_{0}}$ and $p_{z_{0}}$ the perpendicular and longitudinal electron momenta and $R_{g}$ the guiding center radius. $\gamma_{0}$ is the electron relativistic factor, $e$ its charge, and $m_{e}$ its rest mass. $Z_{0}$ is the vacuum impedance and $I_{b}$ the electron beam current. The term $\delta=\frac{s}{2} \frac{d}{d \hat{z}} \ln \left(B_{0}\right)$ accounts for the longitudinal variation of the external magnetic field $B_{0}$ and $C_{m p}=\frac{\pi}{2}\left(\nu_{m p}^{2}-m^{2}\right) J_{m}^{2}\left(\nu_{m p}\right)$ is the geometrical coupling factor.

The boundary conditions imposed for the two moments $\pi_{1}$ and $\pi_{2}$ are homogeneous Dirichlet boundary conditions while radiation boundary conditions are imposed for the field $F$ :

$$
\begin{aligned}
& \pi_{1}\left(\hat{z}_{\text {in }}, \Omega\right)=0 \quad \pi_{2}\left(\hat{z}_{\text {in }}, \Omega\right)=0 \\
& \left.\frac{\mathrm{d} F}{\mathrm{~d} \hat{z}}\right|_{\hat{z}_{\text {in } / \text { ut }}}=\mp i \frac{k_{\|}}{k_{0}} F\left(\hat{z}_{\text {in/out }}, \Omega\right),
\end{aligned}
$$

with $\hat{z}_{\text {in }}, \hat{z}_{\text {out }}$ defining the left and right extremities of the considered interaction space.

Based on a finite difference discretization, this set of equation is solved as a generalized eigenvalue problem $A X=\Omega B X$, with $X$ the eigenvector composed by the two moments $\pi_{1}, \pi_{2}$ and the field $F$ and $\Omega$ the complex eigenfrequency. The eigenvalue problem is solved using the iterative method available from the ARPACK [19] library. Ideally, for one specific operating point, only one execution of this method would be sufficient to obtain all the eigenvalues $\Omega$ of interest related to the different longitudinal $\mathrm{TE}_{m, p, q}$ modes, with $q$ the longitudinal mode number. This is the case in the usual cavity simulations for the TE modes excited close to their cutoff frequency, corresponding to low values of $q$ [17]. As will be shown later, the parasitic oscillation that can be excited in the beam duct or spacer region are Doppler shifted $\mathrm{TE}_{m, p, q}$ modes characterized by large values of $q$. As the method precision is decreasing for larger value of $\Omega$ (assumption $\left|\operatorname{Re}(\Omega) / \omega_{0}\right| \ll 1$ breaks down) [17], the reference frequency $\omega_{0}$ has to be adapted in order to keep $\Omega$ close to zero. This, together with the fact that the longitudinal mode to be excited is unknown, lead to the necessity of scanning a large number of reference frequencies $\omega_{0}$.

The first tests involving a conical interaction region (the part labeled "Beam duct" in figure 1) with radiation boundary conditions at both extremities revealed 
a limitation in our numerical model. The finite difference scheme used was insufficient to simulate this interaction region with a sufficient accuracy. This led us to reformulate the model with a finite element method (FEM). The numerical approach adopted is in fact based on hybrid FEMs [20]. Due to the two different type of problems in equations (1), including two initial value problems (IVP) for (1a) and (1b) and one boundary value problem (BVP) for (1c), a hybrid FEM scheme has to be used. Indeed, using a finite element method formulation based on test functions of the same order as the basis functions for the IVPs leads to numerical instabilities. Using test functions one order lower that the basis functions for Egs. (1a) and (1b), allows to solve this issue. For (1c), a conventional FEM formulation is used. A convergence study is reported in figure 2 for a conical interaction region comparable to a gyrotron smooth-wall beam duct. The three curves are the real part of the frequency $\omega_{r f}$ of a given TE $E_{m, p, q}$ mode in a cylindrical geometry calculated with three different discretization methods. The benefit of using a finite element method of second order is evident.

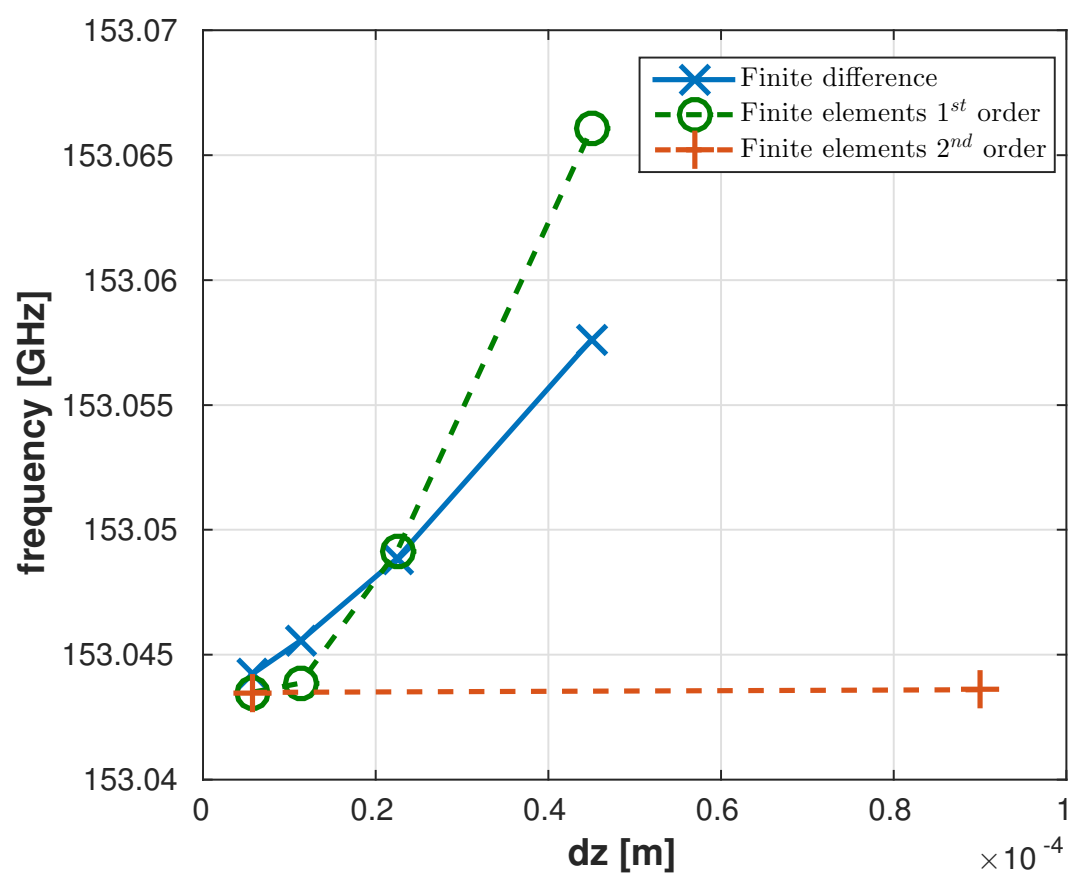

Fig. 2 Frequency of a given $T E_{m, p, q}$ eigenmode calculated with the code TWANGlinspec for different step size for the discretization and for different numerical scheme. The situation treated is a conical structure equivalent to a gyrotron smooth-wall beam duct.

In the spurious instability studies, the most important parameter is their selfexcitation condition, expressed as a minimum starting current. If the minimum 
starting current exceeds the operational gyrotron beam current, the mode would not be excited. The spectral approach is convenient for the computation of starting current. Indeed, as the field is expressed as $e^{i \omega_{r f} t}$, the imaginary part of $\omega_{r f}$ is the wave growth rate. The starting current is the current for which the growth rate is zero. The calculation of the starting current is therefore reduced to a zero-search of $\operatorname{Im}(\Omega)$.

Even though the spectral approach is numerically significantly more efficient than a time evolution approach [17], the overmoded structure of the beam duct, together with the fact that modes could be excited with an important Doppler shift, requires a significant optimization effort. The first measure was to improve the method used for carrying out the zero-search of $\operatorname{Im}(\Omega)$ for the spurious TE modes (many hundreds) by combining a fast adaptive trial and error method giving a coarse first guess for the subsequently applied second method based on either a Newton [21] or Illinois algorithm [22], the combination of both algorithms in case one fails, for instance due to a bad seed, permits to have a reliable outcome. The second measure was to parallelize the code using Message Passing Interface (MPI) for communication between processes. The different parametric scan studies and transverse mode scans that are presented in the next chapter required this parallelization.

The choice of the transverse $\mathrm{TE}_{m, p}$ mode to consider in the simulations is also more intricate in the beam duct simulations than for the normal cavity simulations. This is related to the fact that the choice criteria, the cutoff frequency and the coupling factor, are strongly varying along the interaction region, due to the variation of the beam and wall radii. For these reasons, an extensive scan on all the possible transverse modes is necessary. This scan is in addition to the scan in reference frequency $\omega_{0}$ discussed previously.

The second model used in these studies, TWANG-PIC [23], is a monomode nonlinear particle-in-cell model, based on the common gyro-averaged approach. Contrary to the linear model presented above, the non-linear model includes the electron beam velocity and energy spreads. The code TWANG-PIC has been validated and it has been shown that it is well suited to treat non-stationary regimes $[23,24]$.

\section{Systematic studies}

The strategy considered for the smooth-wall metallic beam duct studies was to start with a simple symmetric situation of a cylindrical constant radius cavity with a downtaper on the gun side and an uptaper on the cavity side as well as an uniform magnetic field. This situation is represented in figure 3 (continuous lines). From this reference, the geometry and the magnetic field profile were progressively adjusted to simulate a real smooth-wall beam duct, represented in dashed lines in figure 3. 


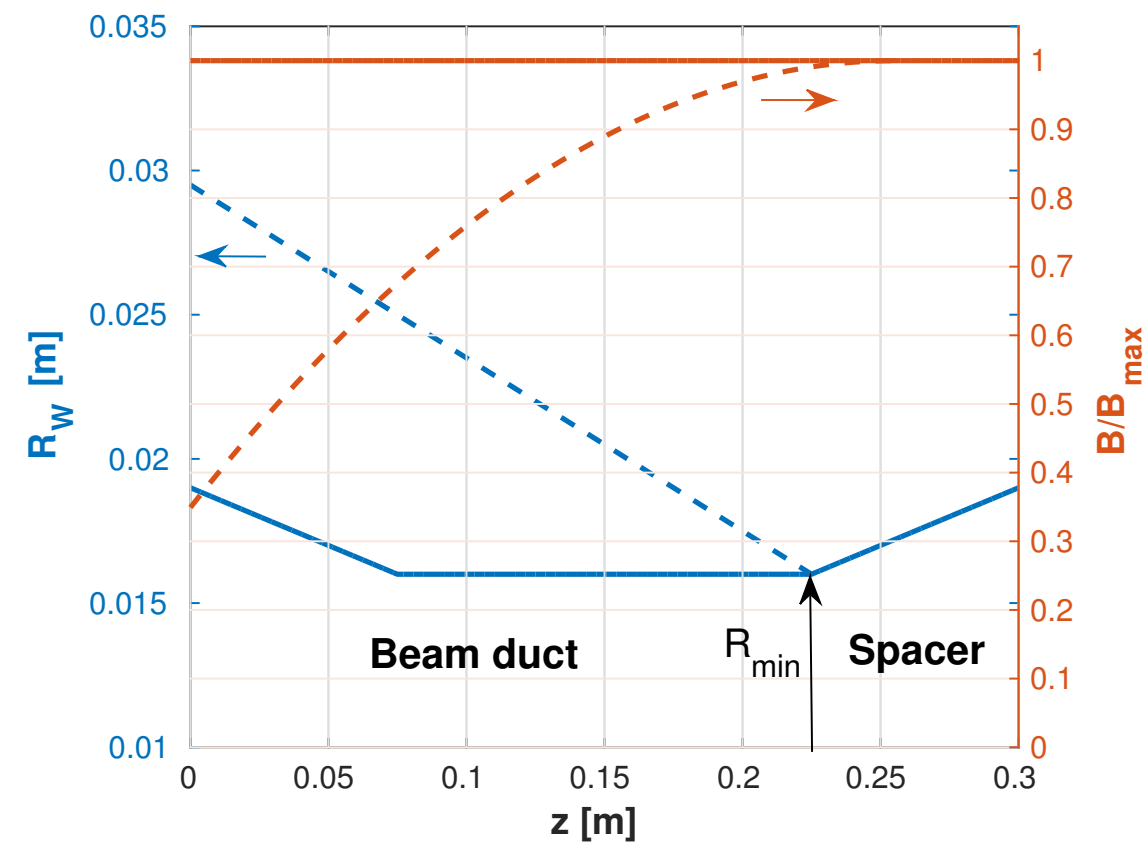

Fig. 3 Beam duct geometry and magnetic field profile (normalized to the maximum value $\left.B_{\max }\right)$. The continuous line corresponds to the reference case. The dashed lines correspond to a more realistic smooth-wall beam duct situation.

For each situation the starting current curves for different transverse mode were calculated. To illustrate the difficulty related to the transverse mode choice, the starting current for four different transverse modes is shown in figure 4 for the reference situation shown with continuous lines in figure 3 for different values of the magnetic field. The continuous line enlightens the minimum starting current for each magnetic field value, thus showing which transverse mode would be excited first. For clarity here, only a reduced selection of transverse modes is shown. The most recent studies involved more than 1400 transverse modes, justifying the choice of MPI code parallelization.

The next step in our study consists in a parametric study, varying the geometry and the magnetic field profile towards a smooth-wall realistic beam duct geometry. As an illustration, the angle $\theta_{1}$ of the beam duct downtaper is changed from the reference situation $\theta_{1}=0^{\circ}$ to a more realistic situation $\theta_{1}=5.1^{\circ}$ or $\theta_{1}=8.5^{\circ}$, as can be seen in figure 5 . Our model neglects the mode conversion, which could be important for a steep wall tapering with larger angle $\theta_{1}$. As will be shown in section IV, even considering these large angles, the mode conversion is moderate. The starting current calculated for the four angles $\theta_{1}$ shown in figure 5 are shown for the mode $T E_{25,3}$ for a range of magnetic field between $4.6 \mathrm{~T}$ and 


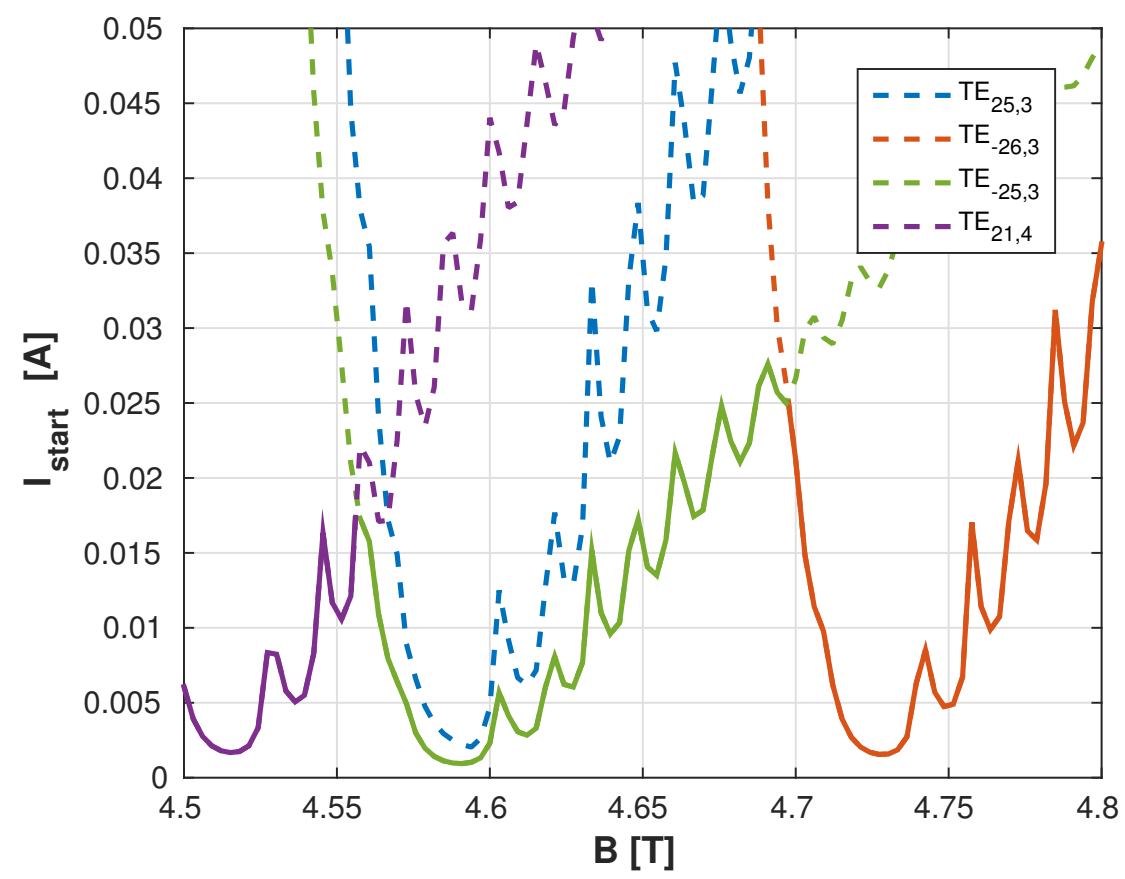

Fig. 4 Starting current calculated with the linear code TWANGlinspec for the situation shown in continuous line in figure 3. For each transverse modes, the different local minima correspond to different longitudinal modes (Parameters: $U_{c}=75 \mathrm{kV}, \alpha=1.3, R_{b}=12 \mathrm{~mm}$ ).

4.9 $\mathrm{T}$ in figure 6 . For this mode, the starting current increases from few $\mathrm{mA}$ for the reference geometry up to $40 \mathrm{~A}$ for the more realistic geometry. Increasing the spacer (see figure 1 ) angle $\theta_{2}$ (as shown in figure 5 ) leads to a similar increase in the starting current. In this case, this would mean that this specific transverse mode $\left(\mathrm{TE}_{25,3}\right)$ is not excited in a typical gyrotron with a nominal beam current around $40 \mathrm{~A}$.

An extensive study was performed as well as for the magnetic field profile. It was shown that the magnetic field profile has less influence on the starting current. On the contrary, for the more realistic geometry situation, a longitudinal translation of the magnetic field profile has a much stronger effect. This could be explained as in the realistic geometry situation, as will be shown later, the interaction is localized in a small region around the minimum radius $R_{\min }$ (see figure 1 ), thus changing the value of the magnetic field profile could have a strong effect on the interaction. 


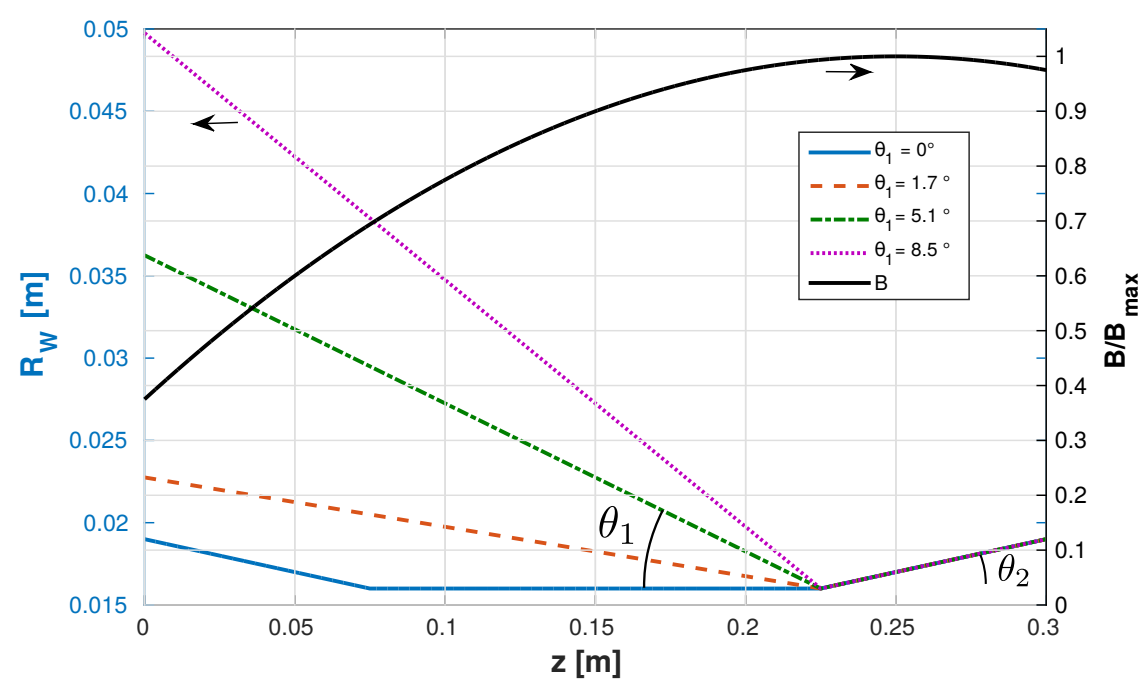

Fig. 5 Beam duct geometry and magnetic field profile. The different geometries considered in figure 6 are shown with dashed lines $\left(\theta_{2}=2.3^{\circ}\right)$.

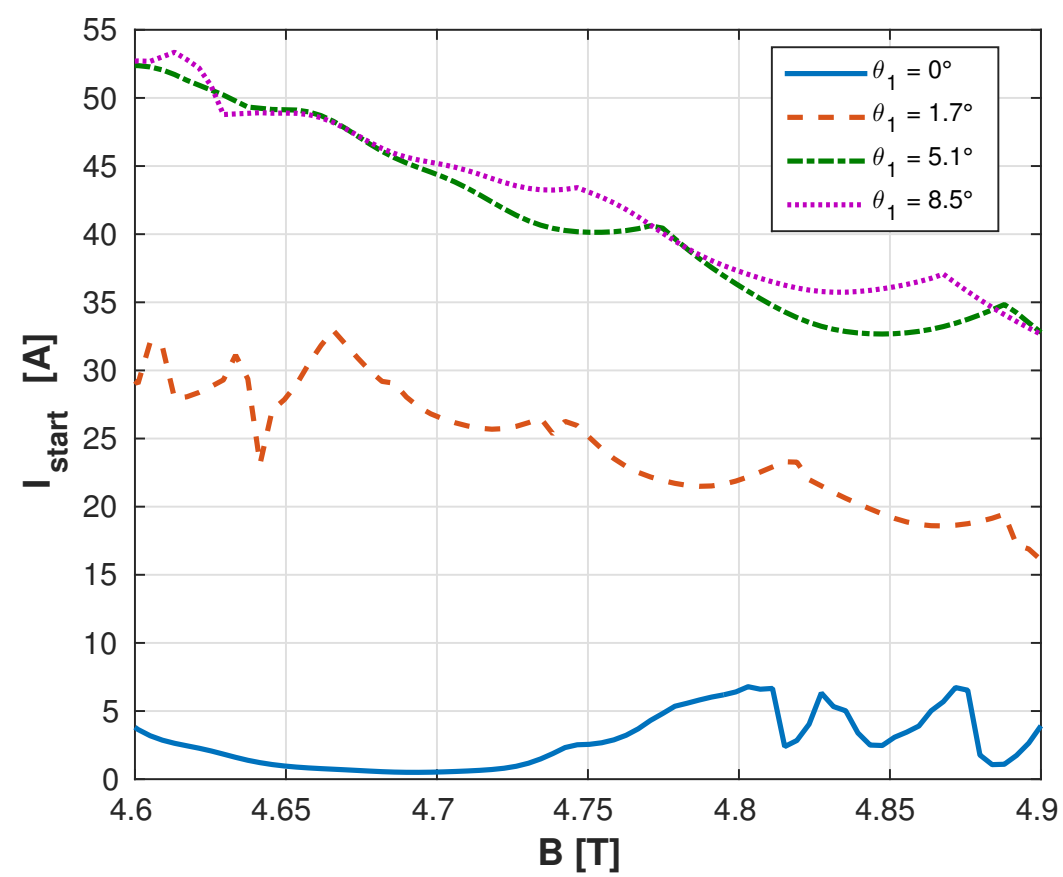

Fig. 6 Starting current of the transverse mode $T E_{25,3}$ for the four different geometries shown in figure 5 and for a range of external magnetic field values. 


\section{Realistic smooth-wall metallic beam duct}

\subsection{Linear simulations}

The systematic studies led to the more realistic situation shown in the figure 7 d). For this case, and considering an electron beam with no velocity spread, the starting current for some transverse modes is still lower than the operating beam current of the gyrotron, meaning that they could be excited. This is the case for example for the mode $\mathrm{TE}_{17,4}$ whose electric field amplitude and phase are represented along the interaction structure in figure $7 \mathrm{a}$ ) and b). For this mode the variation of the rf-field phase, with a negative slope in the left conical section, indicates a backward wave. This is the case for all the potentially unstable modes that were found. As an illustration, the cold cavity (i.e. without electron beam) profile is shown with the green line. This profile is completely different from the self-consistent profile, as usually expected for a backward-wave interaction. As mentioned before, the interaction is very localized in the spacer region, defined in figure 1 . This can be seen in figure $7 \mathrm{c}$ ), where the absolute value of the source term in the wave equation $(1 \mathrm{c})\left(-i C_{3} C_{0} \pi_{1}\right)$ is shown.

The next step is to study a "real" smooth-wall situation. For this the geometry of the dual frequency gyrotron [14] planned on the Tokamak à Configuration Variable (TCV) [15] is chosen. The beam duct implemented in this gyrotron consists of stacked copper and dielectric rings. The very complex non-asymmetric geometry, including strong discontinuities in the wall radius between the rings cannot be simulated with our models. For this reason, a smooth-wall approximation following the wall radius envelope is considered for the beam duct part, while the exact geometry is considered for the spacer, the cavity and its uptaper. The mode conversion due to the wall radius tapering has been evaluated using a scattering matrix model [25], for the geometry shown in figure 1 . With the spurious $\mathrm{TE}_{23,7}$ mode excited in the spacer $\left(\theta=8.5^{\circ}\right)$, the mode conversion in other $T E_{23, n}$ modes is of the order of $15 \%$.

Choosing the high frequency operating point (cavity mode $\mathrm{TE}_{26,7}$ at 126 $\mathrm{GHz}$ ), whose parameters are reported in table 1 and scanning over more than 1400 transverse TE modes, 328 transverse TE modes are found to have a starting current below 40A, the nominal operating beam current. $91 \%$ of these modes are backward waves and around $70 \%$ have their electric field maximum situated in the spacer region as indicated in figure 8 . The starting currents and frequencies of these 328 modes are shown in figures 9 and 10. The modes excited in the spacer region are selected and shown in figure 9. All these modes are gyro-backward modes. Most of the modes below $100 \mathrm{GHz}$ have a frequency higher than their cutoff frequency at the minimum radius $R_{\min }$. They are thus able to propagate through the beam duct while the others are reflected back to the spacer/cavity region. The modes excited after the spacer, in the cavity region, are shown in figure 10 . Both forward and backward propagating mode can be found. The 

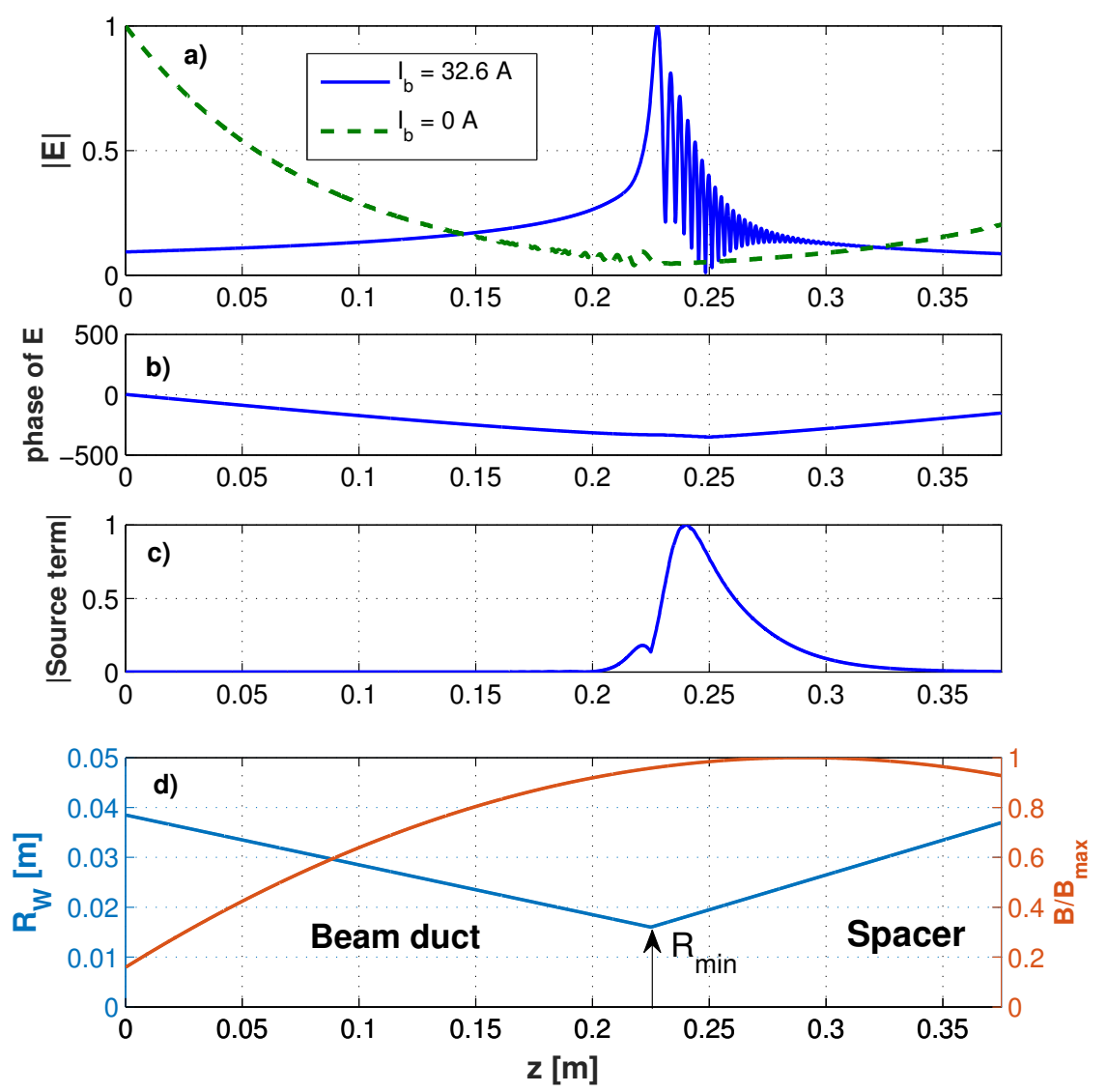

Fig. 7 a) and b) Amplitude and phase of the electric field profile for a beam current slightly exceeding the mode $\left(T E_{17,4}\right)$ starting current. c) Profile of the amplitude of the wave equation source term (1c). d) Profiles of the beam-duct and magnetic field profile. (Parameters: $U_{c}=75$ $\left.\mathrm{kV}, \alpha=1.3, R_{b}=10 \mathrm{~mm}, B_{\max }=4.7 \mathrm{~T}\right)$.

mode indicated with the red circle is the gyrotron operating mode $\mathrm{TE}_{26,7}$ at 126 $\mathrm{GHz}$.

As an illustration, the electric field amplitude and phase for two transverse modes excited in the spacer, the $\mathrm{TE}_{16,4}$ (at $100.7 \mathrm{GHz}$ ) and $\mathrm{TE}_{19,4}$ (at 102.7 $\mathrm{GHz}$ ) are shown in figure 11 for a beam current slightly exceeding their starting current and for the parameters reported in the table $1\left(I_{\text {start }}\left(T_{16,4}\right)=7.5 \mathrm{~A}\right.$, $\left.I_{\text {start }}\left(T E_{20,4}\right)=22.4 \mathrm{~A}\right)$. Both modes have their electric field peaked in the spacer region and exhibit an oscillation with a large longitudinal wave number in the spacer/cavity region. These are backward waves but only the $T E_{16,4}$ is propagating in the beam duct. This is due to the fact that its frequency is higher than the cutoff frequency at the minimum radius $\left(f_{r f}=100.7 \mathrm{GHz}>f_{c o}\left(r_{\min }\right)=100.3\right.$ 
$\mathrm{GHz}$ ). On the contrary, the frequency of the mode $\mathrm{TE}_{20,4}$ is lower than the cutoff frequency $\left(f_{r f}=102.7 \mathrm{GHz}<f_{c o}\left(r_{\min }\right)=111.8 \mathrm{GHz}\right)$ and is thus reflected back towards the cavity. Within the assumption of this model (no velocity spread), even though these modes are excited in the spacer region, they are still interacting with the beam in the cavity region.

Qualitatively consistent with this observation, experimentally, damages on the last rings close to the spacer have been observed [4]. They are indications that parasitic instabilities, if excited, have the maximum rf-field in this region.

\begin{tabular}{|c|c|}
\hline Parameters & Value \\
\hline Magnetic field maximum & $4.98 \mathrm{~T}$ \\
Cathode voltage & $78 \mathrm{kV}$ \\
Pitch angle (no pitch angle spread) & 1.3 \\
Wall conductivity & $1.45 \cdot 10^{7} \mathrm{~S} / \mathrm{m}$ \\
Beam radius & $10.48 \mathrm{~mm}$ \\
TE modes considered & TE $m, n$ with $m \in[-35,35]$ \\
& and $n \in[1,20]$ \\
\hline
\end{tabular}

Table 1 Parameters used for the starting current calculations.
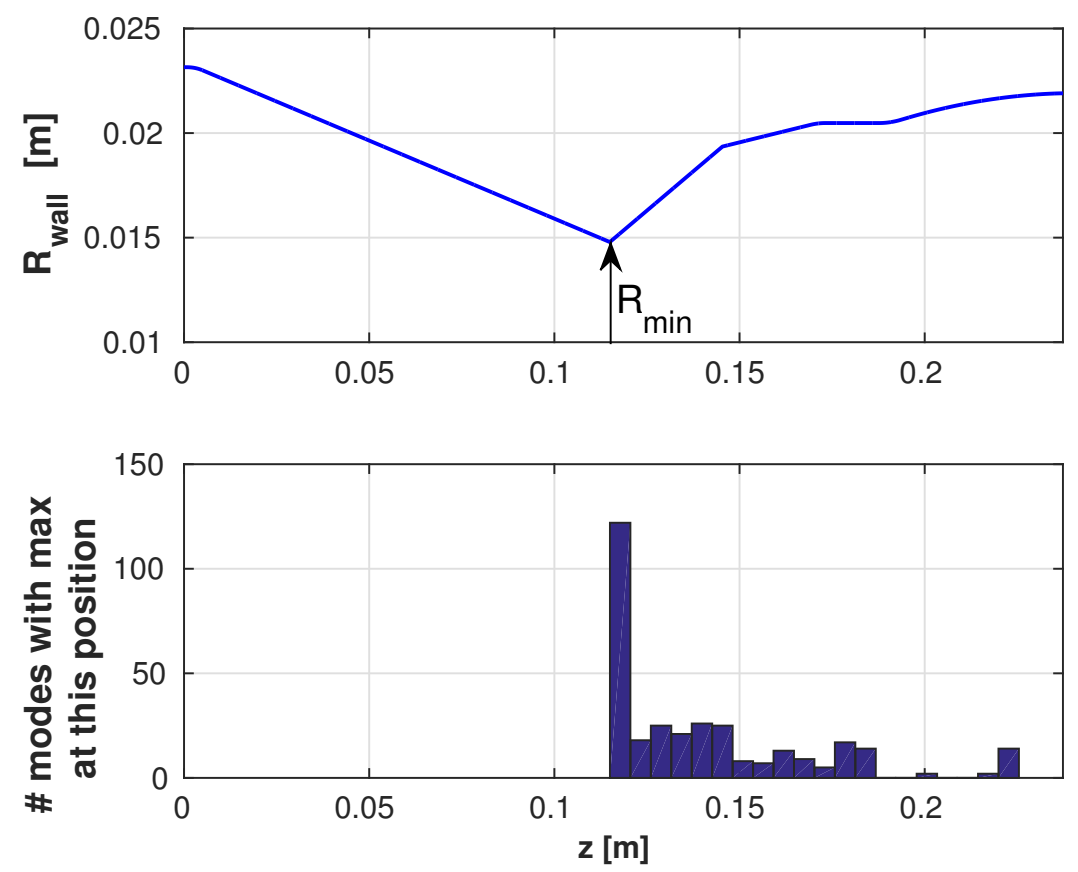

Fig. 8 a) Geometry of the interaction region. b) Histogram representing the position of the electric field maximum for all the transverse modes with $I_{\text {start }}<40 \mathrm{~A}$. 


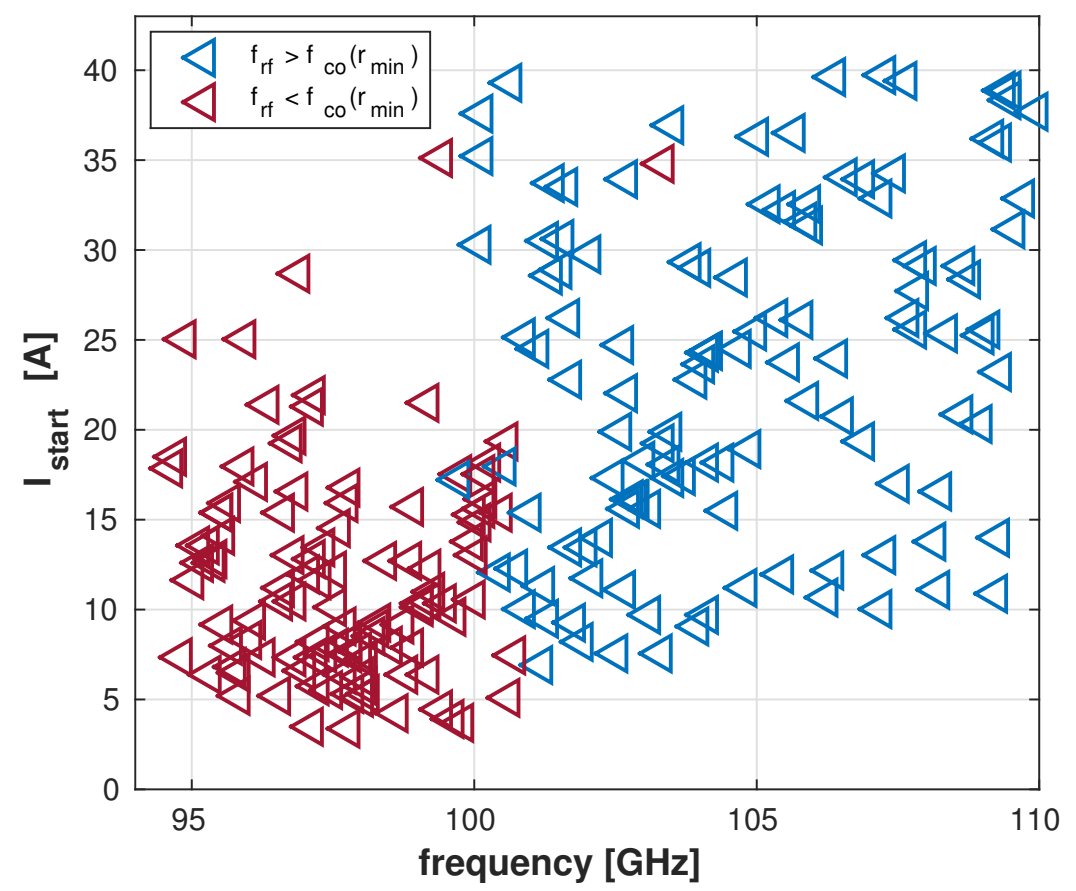

Fig. 9 Starting current and frequency of the modes excited in the spacer region. All these modes are gyro-backward modes. Almost all the modes below $100 \mathrm{GHz}$ (indicated in red) have a frequency higher than the mode cutoff frequency at the minimum radius, meaning that they propagate towards the beam duct direction. The other are reflected towards the gyrotron cavity direction.

\subsection{Nonlinear simulations}

To evaluate the rf-power of these parasitic oscillations and their dependence on the electron beam velocity and energy spread, the monomode nonlinear code TWANGPIC was used on a selection of parasitic modes. The simulations predict that the rf-power generated by these parasitic modes is often exceeding $50 \mathrm{~kW}$. Such rf-power level from parasitic oscillations was never observed experimentally.

Compared to a typical gyrotron mode excited in the cavity of a gyrotron, the parasitic mode excited in the spacer region as shown in figure 11 have an important parallel wave-vector component $k_{\|}$. This can be directly deduced from the fast longitudinal oscillation of their electric field in the spacer plus cavity region. Recalling that the resonant frequency of the wave is given by

$$
\omega_{\mathrm{rf}}=\frac{\Omega_{c}}{\gamma}+k_{\|} v_{\|},
$$




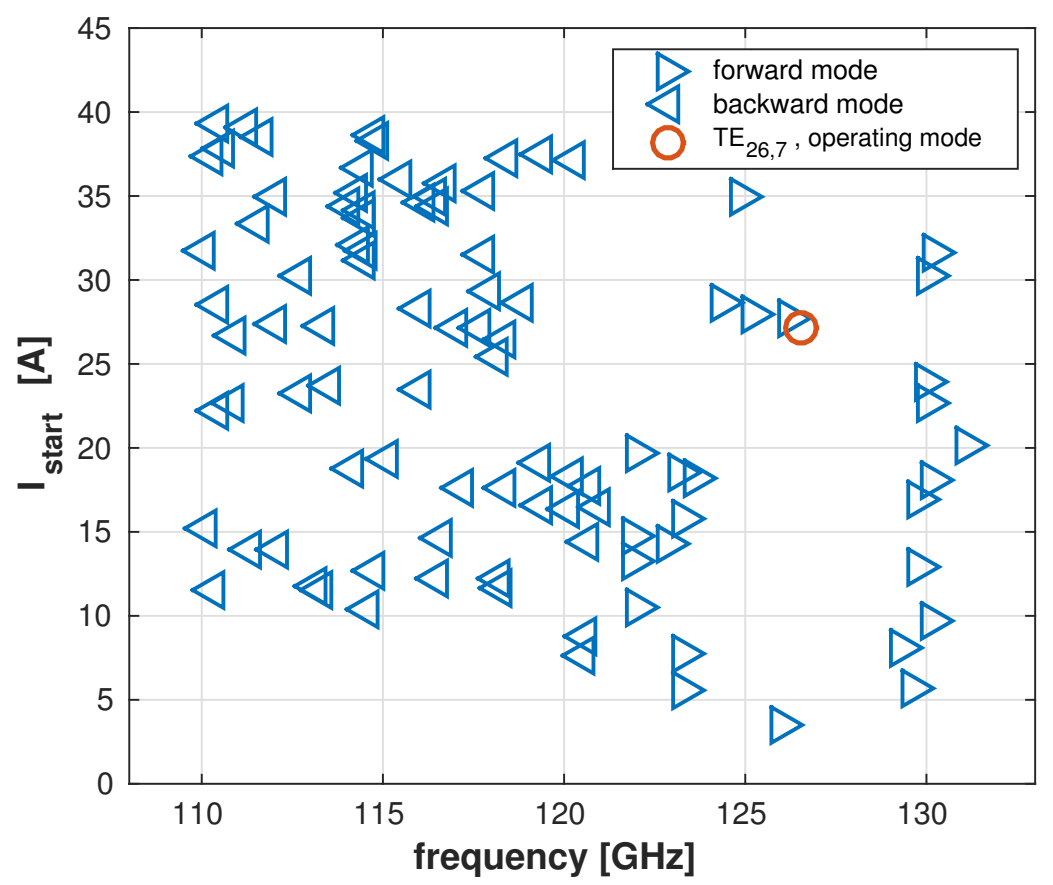

Fig. 10 Starting current and frequency of the modes excited in the cavity region. The direction of the arrow indicate the propagation direction. All these modes have a frequency lower than the mode cutoff frequency at the minimum radius, meaning that the backward propagating modes are reflected towards the gyrotron cavity direction. The operating mode $\mathrm{TE}_{26,7}$ is shown with the red circle.

with $\Omega_{c}$ the cyclotron frequency, $\gamma$ the Lorentz factor and $v_{\|}$the electron parallel velocity. Because of the non-negligible parallel wave-vector, the Doppler shift term could have some importance. It suggests that these modes are more sensitive to an electron velocity spread than a normal cavity mode for which $k_{\|}$is very small. This was verified by using the nonlinear code TWANGPIC to simulate the parasitic mode $\mathrm{TE}_{16,4}$ and the operating mode $T E_{26,7}$ for the same interaction region as in figure $11 \mathrm{c})$, for nominal electron beam parameters $\left(I_{b}=40 \mathrm{~A}\right.$, $\alpha=v_{\perp} / v_{\|}=1.3$ in the cavity) and for different values of the electron pitch angle spread (no energy spread) at the entry of the interaction region $\left(z_{\text {in }}=0\right)$. The rf-power generated for four different spreads in the pitch angle $\alpha$ is shown in figure 12. As expected, due to the large upshift for the parasitic mode, the rf power is much more affected by the velocity spread than the operating mode. For a relative rms spread of 3.8\% (a typical pitch angle spread in electron gun designs) the parasitic mode is no more excited while the operating mode power is reduced by only $3 \%$.

This strong dependency on velocity spread was also observed with the nonlinear and multimode code EURIDICE [26]. A multimode model permits also to 

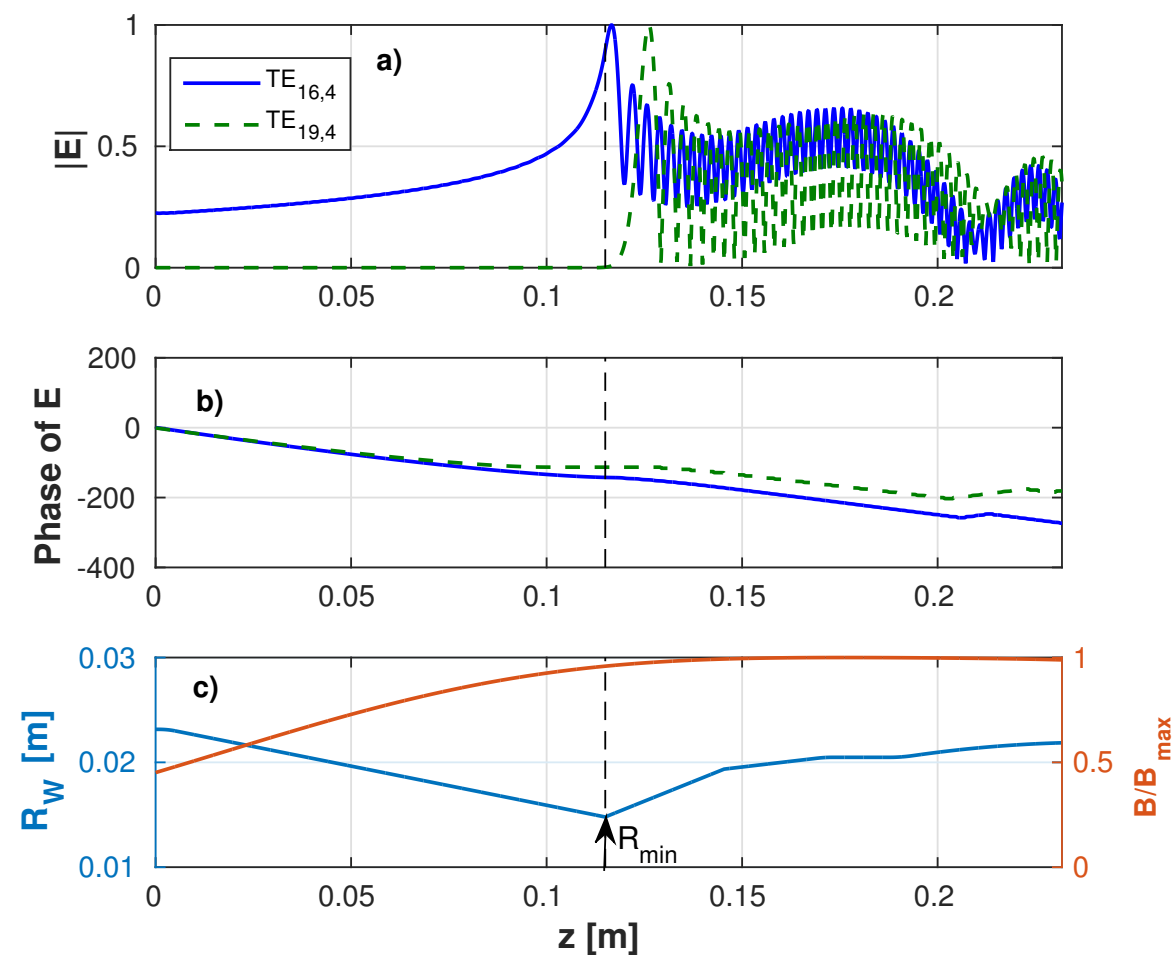

Fig. 11 a) Amplitude of the electric field profile for two transverse modes for a current slightly exceeding their starting current. b) Phase of the electric field profile. c) Interaction space and magnetic field profiles.

potentially fully solve the problem including the whole interaction and considering both the parasitic mode excited in the spacer and the cavity modes in a single simulation. The simulations with EURIDICE showed that the mode competition plays an important role and that only one of the parasitic mode is excited along with the operating mode. This surviving mode is part of the most unstable (lowest starting current) modes calculated with TWANGlinspec. However, some questions still need to be investigated, such as the choice of transverse modes to include in the multimode simulations, which have an impact on the simulation results.

The much larger velocity spread dependency of the parasitic, compared to the cavity modes indicates that the linear model, TWANGlinspec, neglecting this spread is underestimating the minimum starting current compared to the case in which the velocity spread would be taken into account. Based on this consideration, if for some beam duct geometry and properties, using TWANGlinspec, the minimum starting current of all possible TE modes would be above the nominal 
operating current, than this would be even more valid when velocity spread would be included.

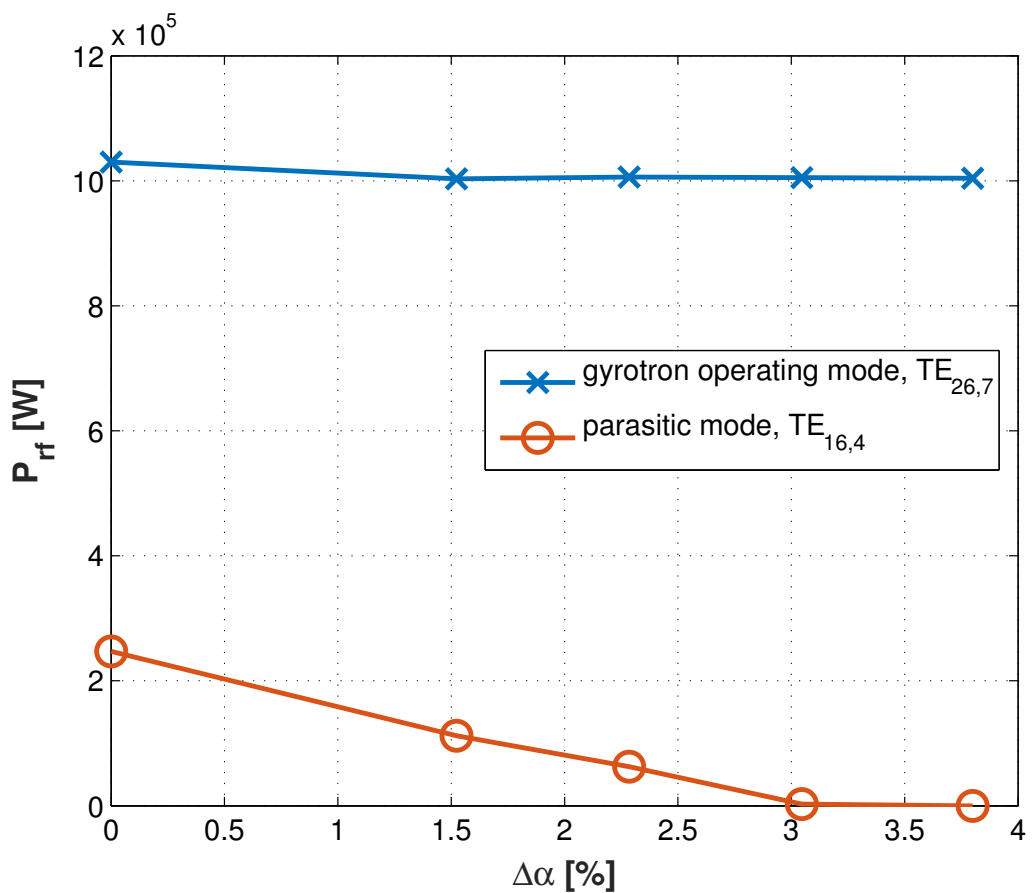

Fig. 12 Rf-power of the nominal $\mathrm{TE}_{26,7}$ and the parasitic $\mathrm{TE}_{16,4}$ modes calculated with the monomode nonlinear code TWANGPIC for different pitch factor spread. The interaction region used is the extended beam duct/spacer/cavity region.

\section{Distributed losses}

Still considering a smooth-wall beam duct, the objective is to study the dependency of the minimum starting current on the wall conductivity, and also to identify in which part of the beam duct plus spacer region the parasitic mode excitation takes place. This was considered for example in $[10,12]$, but in particular for [12] the spacer region was neglected. TWANGlinspec has been validated by reproducing the results presented in [12] with a good qualitative agreement.

Concentrating on a parasitic mode excited in the spacer, the effect of the distribution of the ohmic losses is studied. Two different wall resistivity distributions are represented in figure 13 . The transition between two different resistivity regions is made smoothly to minimize reflections. The two profiles $\rho_{1}$ and $\rho_{2}$ in figure 13 are the two cases where the lossy part is either in the beam-duct part 
for $\rho_{1}$ and in the spacer part for $\rho_{2}$. The starting currents calculated for the transverse mode $T E_{16,4}$ for different values of the resistivity and for these two different profiles are shown in figure 14. For the first profile, there is only a small starting current variation by increasing the resistivity in the beam duct part. On the contrary, the variation is significantly more important if the resistive part is in the spacer region.
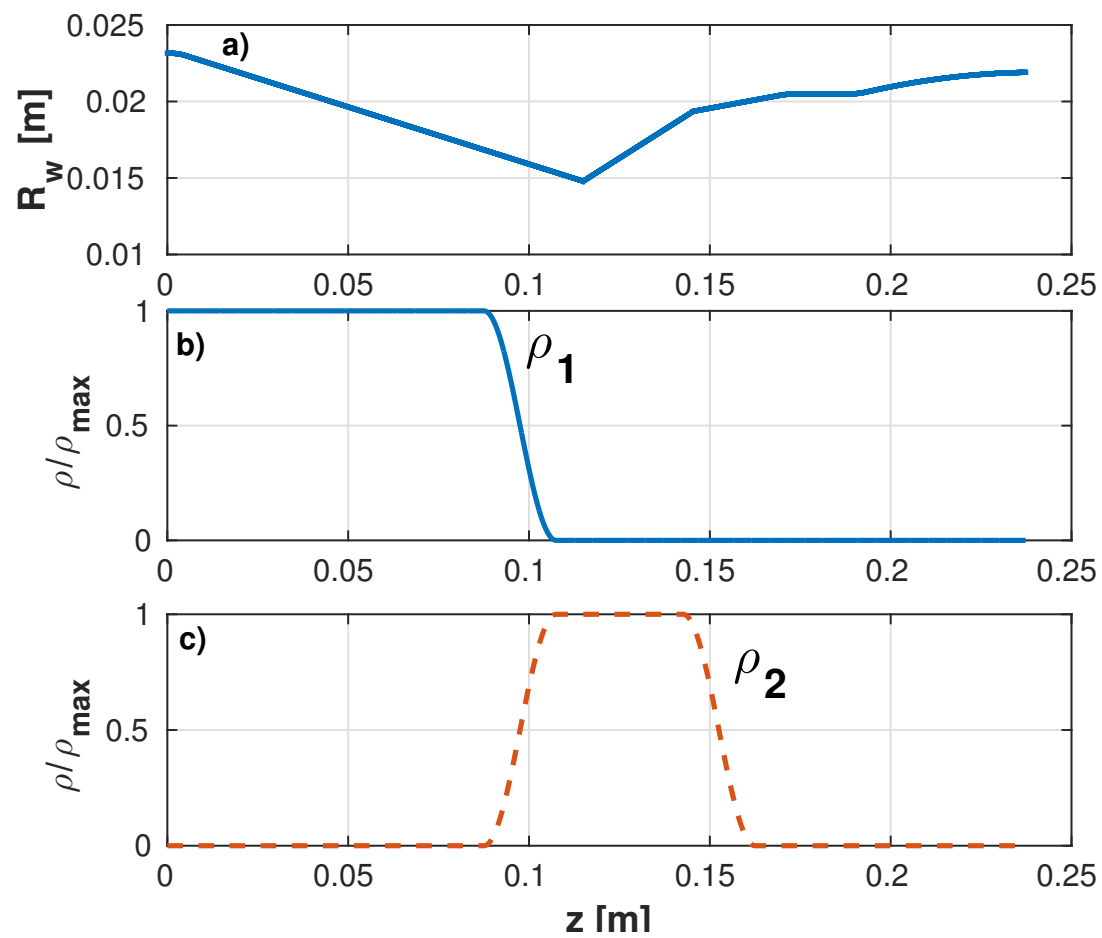

Fig. 13 a) Geometry considered. b) and c) Resistivity profiles considered for the starting current study in figure 14

As the nominal operating beam current is $40 \mathrm{~A}$, one could see that with the wall resisitivies considered in figure 14 and with no velocity spread, the $T E_{16,4}$ mode is still expected to be excited as the largest starting current is around 21 A. However, for this resistivity value, the good conductor assumption made in the derivation of the surface impedance model is violated $\left(\sqrt{\frac{\sigma}{\omega \epsilon_{0}}} \gg 1\right)$ [7].

To study the effect of a lossy dielectric smooth-wall beam duct of finite thickness [2] requires to solve the dispersion relation for $\mathrm{HE}_{m n}$ and $\mathrm{EH}_{m n}$ modes [13]. This is being implemented in TWANGlinspec and will be part of a future publication. 


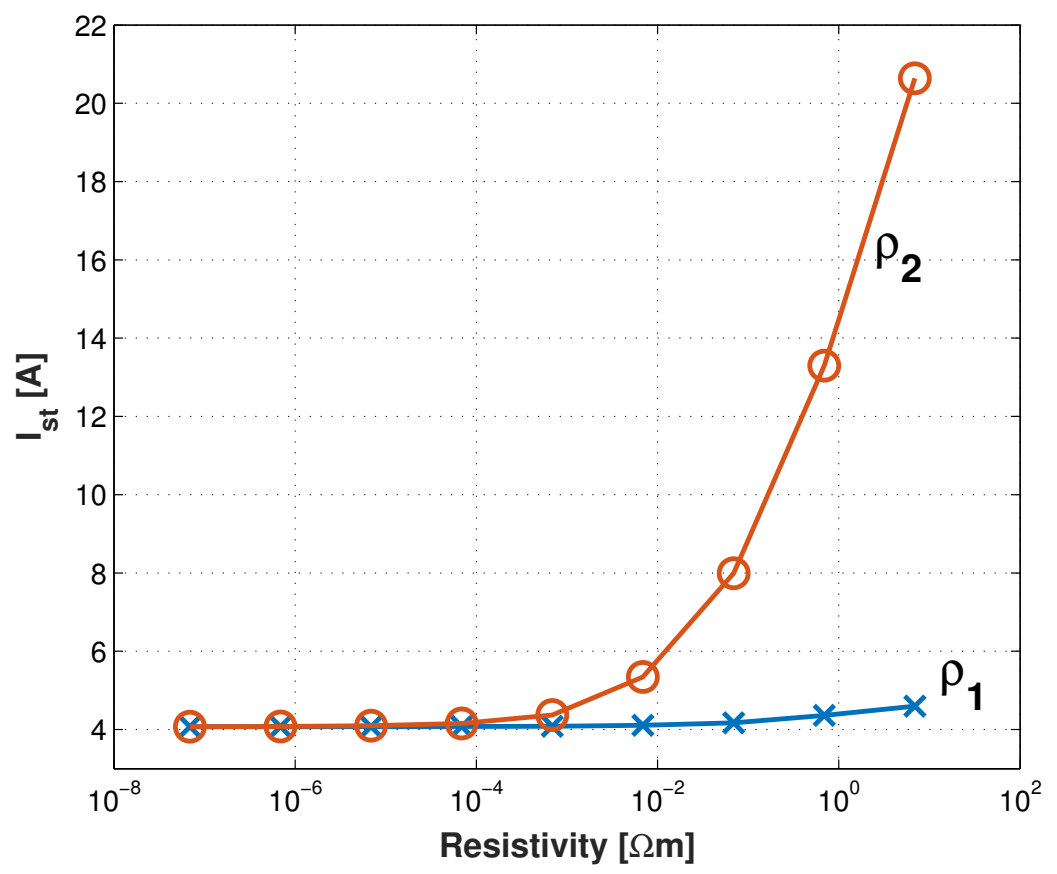

Fig. 14 Starting current calculated for the transverse mode $T E_{16,4}$ by varying the wall resistivity and for the two different resistivity distributions shown in figure 13 . The resistivity of pure copper is $1.7 \cdot 10^{-8} \Omega \mathrm{m}$.

\section{Conclusion}

The recently developed self-consistent linear and spectral code TWANGlinspec has been adapted to treat oscillations that could occur in the extended region upstream to the cavity. The strong spatial inhomogeneities in the wall radius and magnetic field profile compared to the cavity region required a new formulation using a hybrid finite element method scheme. The code has been parallelized using MPI, allowing to efficiently treat parameter scans for a large number of TE modes. The strategy used to study the beam duct instabilities was to start from a simple reference situation and varying progressively the geometry and the magnetic field profile to approach a realistic situation. This shows that the tapering angles of the beam duct and the spacer are found to be the pivotal parameters impacting strongly the starting current of the parasitic modes. Due to the localized interaction region of the parasitic modes, a translation of the magnetic field profile along the longitudinal direction has also a strong influence on the self-excitation of any given modes. However, considering the large number of potentially unstable modes, when the minimum starting current of a specific mode increased, the minimum starting current of a different mode is reduced. 
For a realistic situation an important difficulty arises with the choice of the transverse modes to consider. In the well known cavity interaction where the wall radius and the magnetic field profile vary slowly, the mode to consider are conveniently chosen with respect to their frequency and their geometrical coupling factor. In the beam duct region involving strong spatial inhomogeneities, the same selection criteria cannot be used and an extensive scan over all possible transverse modes has to be considered. These scans show that the spacer region, situated between the beam duct and the cavity plays an important role in the excitation of Doppler shifted gyro-backward wave modes. These instabilities are peaked in the spacer region but continue to interact with the electron beam throughout the cavity.

Non-linear simulations including a realistic electron beam with spread indicate that these parasitic oscillations, unlike the gyrotron operating mode, depend strongly on the velocity spread of the electron beam. This could explain why such parasitic oscillations with a rf-power exceeding $50 \mathrm{~kW}$ were never observed experimentally.

This leads us to the conclusion that the model TWANGlinspec, neglecting the velocity spread, is underestimating the minimum starting current. Based on that, a situation where TWANGlinspec would not predict the excitation of any modes could be considered as a safe, free of instabilities situation in a realistic case with a non ideal electron beam.

A solution to damp these oscillations is to add a dielectric coating in the end of the beam duct and in the spacer region. This has been approximated via a surface impedance boundary condition, as it is commonly done to evaluate ohmic losses in the walls of gyrotron of amplifiers $[11,12]$. As expected from the interaction localized in the spacer, an ohmic losses distribution study shows that it is more effective to put the lossy material in the spacer region. However, the good conductor assumption made by the model is approaching its limit for wall conductivity capable of stabilizing the instabilities. A self-consistent simulation including a lossy dielectric coating of finite thickness requires an adaptation of the model and will be part of a future publication.

Acknowledgements We dedicate this article to our late friend T.M. Tran. This work was supported in part by the Swiss National Science Foundation, by the EUROfusion WPHCD programme and by Fusion for Energy under Grants F4E-GRT-432 and F4E-GRT-553 to the European Gyrotron Consortium (EGYC). EGYC is a collaboration among SPC, Switzerland; KIT, Germany; HELLAS, Greece; IFP-CNR, Italy. This work has been carried out within the framework of the EUROfusion Consortium and has received funding from the Euratom research and training programme 2014-2018 under grant agreement No 633053. The views and opinions expressed herein are the sole responsibility of the authors and do not necessarily reflect the views of the European Commission and F4E. The author would like to thank D. Wagner and S. Brunner for precious scientific discussions. 


\section{References}

1. I. I. Antakov, I. G. Gachev, and E. V. Zasypkin. Self-excitation of spurious oscillations in the drift region of gyrotrons and their influence on gyrotron operation. IEEE Transactions on Plasma Science, 22(5):878-882, October 1994.

2. K Sakamoto, A Kasugai, Y Ikeda, K Hayashi, K Takahashi, S Moriyama, M Seki, T Kariya, Y Mitsunaka, T Fujii, and T Imai. Development of 170 and $110 \mathrm{GHz}$ gyrotrons for fusion devices. Nuclear Fusion, 43(8):729-737, August 2003.

3. M. Pedrozzi, S. Alberti, J. P. Hogge, M. Q. Tran, and T. M. Tran. Electron beam instabilities in gyrotron beam tunnels. Physics of Plasmas, 5(6):2421-2430, June 1998.

4. G. Gantenbein, G. Dammertz, J. Flamm, S. Illy, S. Kern, G. Latsas, B. Piosczyk, T. Rzesnicki, A. Samartsev, A. Schlaich, M. Thumm, and I. Tigelis. Experimental Investigations and Analysis of Parasitic RF Oscillations in High-Power Gyrotrons. IEEE Transactions on Plasma Science, 38(6):1168-1177, June 2010.

5. G. G. Denisov, V. E. Zapevalov, A. G. Litvak, and V. E. Myasnikov. Megawatt Gyrotrons for ECR Heating and Current-Drive Systems in Controlled-Fusion Facilities. Radiophysics and Quantum Electronics, 46(10):757-768, October 2003.

6. M. Blank, K. Felch, P. Borchard, P. Cahalan, S. R. Cauffman, Tak Sum Chu, and H. Jory. Demonstration of a high-power long-pulse $140-\mathrm{GHz}$ gyrotron oscillator. IEEE Transactions on Plasma Science, 32(3):867-876, June 2004.

7. I. G. Chelis, K. A. Avramidis, Z. C. Ioannidis, and I. G. Tigelis. Improved Suppression of Parasitic Oscillations in Gyrotron Beam Tunnels by Proper Selection of the Lossy Ceramic Material. IEEE Transactions on Electron Devices, 65(6):2301-2307, June 2018.

8. M. Garven, J. P. Calame, B. G. Danly, K. T. Nguyen, B. Levush, F. N. Wood, and D. E. Pershing. A gyrotron-traveling-wave tube amplifier experiment with a ceramic loaded interaction region. IEEE Transactions on Plasma Science, 30(3):885-893, June 2002.

9. I. G. Tigelis, J. L. Vomvoridis, and S. Tzima. High-frequency electromagnetic modes in a dielectric-ring loaded beam tunnel. IEEE Transactions on Plasma Science, 26(3):922-930, June 1998.

10. Kwo Ray Chu, Han-Ying Chen, Chien-Lung Hung, Tsun-Hsu Chang, L.R. Barnett, ShihHung Chen, Tz-Te Yang, and D.J. Dialetis. Theory and experiment of ultrahigh-gain gyrotron traveling wave amplifier. IEEE Transactions on Plasma Science, 27(2):391-404, April 1999.

11. K. R. Chu, L. R. Barnett, H. Y. Chen, S. H. Chen, Ch. Wang, Y. S. Yeh, Y. C. Tsai, T. T. Yang, and T. Y. Dawn. Stabilization of Absolute Instabilities in the Gyrotron Traveling Wave Amplifier. Physical Review Letters, 74(7):1103-1106, February 1995.

12. Jiao Yu, Thomas M. Antonsen, and Gregory S. Nusinovich. Excitation of Backward Waves in Beam Tunnels of High-Power Gyrotrons. IEEE Transactions on Plasma Science, 38(6):1193-1199, June 2010.

13. Chao-Hai Du and Pu-Kun Liu. Millimeter-Wave Gyrotron Traveling-Wave Tube Amplifiers. Springer Berlin Heidelberg, Berlin, Heidelberg, 2014.

14. S. Alberti, F. Braunmueller, J. Genoud, J. P. Hogge, T. M. Tran, M. Q. Tran, K. Avramidis, I. G. Pagonakis, J. Jin, S. Illy, G. Gantenbein, J. Jelonnek, and F. Cismondi. Dual-frequency, $126 / 84 \mathrm{GHz}, 1 \mathrm{MW}$ gyrotron for the upgrade of the TCV EC-system. In 2015 40th International Conference on Infrared, Millimeter, and Terahertz waves (IRMMW-THz), pages 1-2, August 2015

15. S. Coda et al. Overview of the TCV tokamak program: scientific progress and facility upgrades. Nuclear Fusion, 57(10):102011, October 2017.

16. S. Alberti, T. M. Tran, K. A. Avramides, F. Li, and J.-P. Hogge. Gyrotron parasiticeffects studies using the time-dependent self-consistent monomode code TWANG. 2011 36th International Conference on Infrared, Millimeter, and Terahertz Waves (irmmw-Thz), 2011.

17. J. Genoud, T. M. Tran, S. Alberti, F. Braunmueller, J.-Ph Hogge, M. Q. Tran, W. C. Guss, and R. J. Temkin. Novel linear analysis for a gyrotron oscillator based on a spectral approach. Physics of Plasmas (1994-present), 23(4):043101, April 2016. 
18. F. Braunmueller, T. M. Tran, S. Alberti, J.-Ph Hogge, and M. Q. Tran. Moment-based, self-consistent linear analysis of gyrotron oscillators. Physics of Plasmas, 21(4):043105, April 2014.

19. D.C. Sorensen, R.B. Lehoucq, C. Yang, and K. Maschhoff. ARPACK - Arnoldi Package, 1996.

20. Franco Brezzi and Michel Fortin, editors. Mixed and Hybrid Finite Element Methods, volume 15 of Springer Series in Computational Mathematics. Springer New York, New York, NY, 1991.

21. William H. Press, Brian P. Flannery, Saul A. Teukolsky, and William T. Vetterling. Numerical Recipes: The art of scientific computing. Cambridge Univ. Press, 1986.

22. Germund Dahlquist and ke Bjrck. Numerical Methods. Courier Corporation, 2003.

23. F. Braunmueller, T. M. Tran, Q. Vuillemin, S. Alberti, J. Genoud, J.-Ph Hogge, and M. Q. Tran. TWANG-PIC, a novel gyro-averaged one-dimensional particle-in-cell code for interpretation of gyrotron experiments. Physics of Plasmas (1994-present), 22(6):063115, June 2015.

24. S. Alberti, T. M. Tran, S. Brunner, F. Braunmueller, J. Genoud, J.-Ph Hogge, and M. Q. Tran. Generalized Radiation Boundary Conditions in Gyrotron Oscillator Modeling. Journal of Infrared, Millimeter, and Terahertz Waves, pages 1-16, September 2015.

25. D. Wagner, M. Thumm, G. Gantenbein, W. Kasparek, and T. Idehara. Analysis of a Complete Gyrotron Oscillator Using the Scattering Matrix Description. International Journal of Infrared and Millimeter Waves, 19(2):185-194, February 1998.

26. K. A. Avramides, I. Gr Pagonakis, C. T. latrou, and J. L. Vomvoridis. EURIDICE: A code-package for gyrotron interaction simulations and cavity design. In E. Westerhof and Pwjm Nuij, editors, Ec-17 - 17th Joint Workshop on Electron Cyclotron Emission and Electron Cyclotron Resonance Heating, volume 32, page 04016. E D P Sciences, Cedex A, 2012. 